Supporting Information for

\title{
Plasmonic Nanozymes: Engineered Gold Nanoparticles Exhibit Tunable Plasmon-Enhanced Peroxidase-Mimicking Activity
}

Yang Zhang, ${ }^{\dagger}$ Esteban Villarreal, Guangfang Grace Li, Wei Wang, ${ }^{\ddagger}$ and Hui Wang*

Department of Chemistry and Biochemistry, University of South Carolina, Columbia, South Carolina 29208, United States

* To whom correspondence should be addressed.

Email:wang344@mailbox.sc.edu (H.Wang); Phone: 1-803-777-2203; Fax: 1-803-777-9521.

Current addresses:

${ }^{\dagger}$ CAS Key Laboratory of Design and Assembly of Functional Nanostructures and Fujian Provincial Key Laboratory of Nanomaterials, Fujian Institute of Research on the Structure of Matter, Chinese Academy of Sciences, Fuzhou, Fujian 350002, China; Xiamen Institute of RareEarth Materials, Haixi Institute, Chinese Academy of Sciences, Xiamen, Fujian 361021, China

${ }^{\star}$ School of Chemistry and Materials Science, Nanjing University of Information Science and Technology, Nanjing, Jiangsu 210044, China 


\section{S1. Experimental Details, Data Analysis, and Additional Discussions}

\section{S1.1 Chemicals and Materials}

Gold(III) chloride trihydrate $\left(\mathrm{HAuCl}_{4} \cdot 3 \mathrm{H}_{2} \mathrm{O}\right.$, ACS grade) was purchased from J.T. Baker. 3,3',5,5'Tetramethylbenzidine (TMB, $1.20 \mathrm{mM}$ ) was purchased from SeraCare. Horseradish peroxidase (HRP, EIA grade) was obtained from Novex. Sodium acetate trihydrate $\left(\mathrm{CH}_{3} \mathrm{COONa} \cdot 3 \mathrm{H}_{2} \mathrm{O}, \mathrm{NaAc}, \mathrm{ACS}\right.$ grade $)$ was obtained from Fisher Chemical. Hydrochloric acid $(\mathrm{HCl}, 37 \%)$, sodium borohydride $\left(\mathrm{NaBH}_{4}, 99 \%\right)$, L-ascorbic acid (AA, 99.5\%), dopamine hydrochloride, tris(hydroxymethyl)aminomethane, and thionine acetate salt were purchased from Sigma-Aldrich. Cetyltrimethylammonium chloride (CTAC, 96\%) was obtained from Alfa Aesar. Iso-propanol (99.5\%, ACS grade) and acetic acid (HAc, 99.7\%, ACS grade) were obtained from Macron. Hydrogen peroxide $\left(\mathrm{H}_{2} \mathrm{O}_{2}, 30 \%\right.$, ACS grade) was obtained from Ward's Science. All reagents were used as received without further purification. Ultrapure water (18.2 M $\Omega$ resistivity, Barnstead EasyPure II 7138) was used for all experiments.

\section{S1.2 Synthesis of Au Nanozymes}

Colloidal Au surface roughened nanoparticles (SRNPs) and quasi-spherical nanoparticles (QSNPs), which served as the peroxidase-mimicking nanozymes, were synthesized using a seed-mediated nanocrystal growth method we previously developed. ${ }^{1-3}$ The CTAC-capped colloidal Au seeds $(\sim 3 \mathrm{~nm}$ in diameter) were first synthesized by reducing $\mathrm{HAuCl}_{4}$ with $\mathrm{NaBH}_{4}$ in the presence of CTAC. Briefly, $0.25 \mathrm{~mL}_{\text {of }} 10 \mathrm{mM} \mathrm{HAuCl}_{4}$ solution was added into $10 \mathrm{~mL}$ of $100 \mathrm{mM} \mathrm{CTAC}$ solution under magnetic stir. Then $0.30 \mathrm{~mL}$ of $10 \mathrm{mM}$ fresh, ice-cold $\mathrm{NaBH}_{4}$ was injected to the mixture. The solution was stirred vigorously for $1 \mathrm{~min}$, then left undisturbed for $2 \mathrm{~h}$, and finally diluted with $100 \mathrm{mM} \mathrm{CTAC}$ by 1000 -fold for future use.

The growth solution for Au SRNPs consisted of $0.50 \mathrm{~mL}$ of $10 \mathrm{mM} \mathrm{HAuCl}_{4}, 0.10 \mathrm{~mL}$ of $100 \mathrm{mM}$ AA, and $10.0 \mathrm{~mL}$ of $100 \mathrm{mM}$ CTAC. The overall particle sizes were controlled by varying the amount of seeds added into the growth solution. The plasmon resonance peak of the colloidal Au SRNPs progressively red-shifted as the overall particle sizes increased. To synthesize Au SRNPs with plasmon resonance peak centered at $785 \mathrm{~nm}$ (denoted as Au-785), $20 \mu \mathrm{L}$ of the seeds was introduced into the growth solution. The amount of the seeds needed for the synthesis of Au SRNPs with plasmon resonance peak centered at 638, 714, 858, and $980 \mathrm{~nm}$ (denoted as Au-638, Au-714, Au-858, and Au980, respectively) were $45 \mu \mathrm{L}, 28 \mu \mathrm{L}, 14 \mu \mathrm{L}$, and $8 \mu \mathrm{L}$, respectively. The mixtures were gently mixed for $30 \mathrm{~s}$, then left undisturbed for $12 \mathrm{~h}$. Au QSNPs were fabricated following a similar protocol except for the addition of $\mathrm{HCl}$. To synthesize Au QSNPs with an optical extinction peak at $\sim 638 \mathrm{~nm}, 0.20 \mathrm{~mL}$ of $1.0 \mathrm{M} \mathrm{HCl}$ was added to the growth solution and $40 \mu \mathrm{L}$ of the Au seeds was introduced into the growth solution. The as-synthesized Au SRNPs and QSNPs were washed with water 3 times before finally being redispersed in water. The particle concentrations of Au SRNPs and QSNPs were calculated based on the amount of seeds used for the particle syntheses following a previously reported procedure. ${ }^{2}$

\section{S1.3 Structural Characterizations of the Au Nanozymes}

The structures of the Au nanozymes were characterized using a Zeiss Ultraplus scanning electron microscope (SEM). The samples for SEM imaging were pre-dispersed in water and drop-dried on silicon wafers. The distributions of particle sizes were obtained based on the SEM images of each 
sample using the Image J software. The transmission electron microscopy (TEM) images were obtained using a Hitachi H-7800 transmission electron microscope, operated at an accelerating voltage of $100 \mathrm{kV}$. Samples for TEM measurements were dispersed in ethanol and drop-dried on 300 mesh carbon-coated $\mathrm{Cu}$ grids (Electron Microscopy Science Inc.). The optical extinction spectra of colloidal Au nanozymes were collected at room temperature using a Beckman Coulter DU-640 spectrophotometer.

\section{S1.4 Cyclic Voltammetry (CV) Results}

The $\mathrm{CV}$ measurements were performed using a $\mathrm{CHI} 660 \mathrm{E}$ workstation $(\mathrm{CH}$ Instruments, Austin, Texas) at room temperature with a standard three-electrode system composed of a saturated calomel electrode (SCE) as the reference, a glassy carbon electrode (GCE, $3.0 \mathrm{~mm}$ diameter) as the working electrode, and a Pt wire as the counter electrode. The GCEs were polished with $0.3 \mathrm{~mm}$ alumina slurry and followed by washing with water and ethanol before use. $5.0 \mu \mathrm{L}$ of colloidal ink containing $9.6 \mathrm{pM}$ $\mathrm{Au}$ QSNPs or SRNPs were dropped and air-dried on the pretreated GCEs at room temperature. Then 2 $\mu \mathrm{L}$ of nafion solution $(0.2 \mathrm{wt} \%)$ was dropped to hold the Au nanoparticles. The $\mathrm{CV}$ measurements were carried out at room temperature in $\mathrm{N}_{2}$-saturated $0.5 \mathrm{M} \mathrm{H}_{2} \mathrm{SO}_{4}$ electrolyte solution at a potential sweep rate of $5.0 \mathrm{mV} \mathrm{s}^{-1}$. The $\mathrm{CV}$ results shown in Figure S2 revealed a strong correlation between the oxidation potential and the coordination number of $\mathrm{Au}$ surface atoms. During the anodic potential sweeps, the Au surface atoms with lower coordination numbers were electrochemically oxidized to form oxide adlayers at lower potentials than those with higher coordination numbers. ${ }^{3-6}$ QSNPs exhibited a major oxidation peak above $1.3 \mathrm{~V}$ ( $v s$. SCE), which was the electrochemical signature of oxide adlayer formation on the $\{100\}$ and $\{111\}$ facets of $\mathrm{Au}$, whereas the oxidation of the undercoordinated $\mathrm{Au}$ atoms on the surfaces of SRNPs occurred at significantly lower potentials ranging from 0.9 to $1.2 \mathrm{~V}$ (vs. SCE). During the subsequent cathodic potential sweeps, the oxide adlayers were electrochemically stripped-off, which was signified by a sharp reduction peak centered around $0.9 \mathrm{~V}$ (vs. SCE). ${ }^{3-6}$ The average electrochemical surface area (ECSA) of each SRNP was calculated to be 4.2 times as large as that of each QSNP based on the relative areas of the oxide stripping peaks. Assuming the specific charge associated with gold oxide stripping to be $450 \mu \mathrm{C} \mathrm{cm}^{-2}, 7$ the specific ECSAs of the SRNPs and the QSNPs were estimated to be $\sim 12.6 \times 10^{4}$ and $3.0 \times 10^{4} \mathrm{~nm}^{2}$ per particle, respectively.

\section{S1.5 Kinetic Measurements}

The kinetic measurements were carried out in a $1 \mathrm{~cm} \times 1 \mathrm{~cm} \times 4 \mathrm{~cm}$ quartz cuvette containing various concentrations of $\mathrm{H}_{2} \mathrm{O}_{2}, \mathrm{TMB}$, and Au nanozymes or HRP in HAc-NaAc buffer. The $\mathrm{pH}$ values of the reaction solutions were adjusted by varying the composition of the buffer solutions (see details in Table S1). The total volume of the reactant mixture was kept at $3.0 \mathrm{~mL}$. To avoid the photothermal heating of the solutions under laser illumination, the colloidal nanozymes were kept at low concentrations below $400 \mathrm{fM}\left(2.4 \times 10^{8}\right.$ particles $\mathrm{mL}^{-1}$, optical densities at the plasmon resonance wavelengths $\left.<0.1\right)$. A temperature-controlled circulating water bath was used to control the temperature of the bulk solutions, and the reactions were conducted under constant magnetic stir $(800 \mathrm{rpm})$. Three continuous-wave diode lasers purchased from Lasever Inc. (Ningbo, Zhejiang, China) with the tunable power output in the range of 0-3.0 W and emission wavelengths at $638 \mathrm{~nm}$ (model no. LSR638CP8), $785 \mathrm{~nm}$ (model no. LSR785NL), and $980 \mathrm{~nm}$ (model no. LSR980NL) were used as the excitation sources. The laser beams were collimated with a $4 \mathrm{~mm} \times 4 \mathrm{~mm}$, square-shaped cross-section and incident vertically from the top surface into the reaction 
solution. The reaction progress was monitored based on the temporal evolution of the concentration of oxidized TMB (charge-transfer complex exhibiting a characteristic blue color, denoted as oxTMB) quantified by UV-visible-near infrared absorption spectroscopy using a Beckman Coulter Du 640 spectrophotometer at certain time intervals. The UV-visible-near infrared absorption spectra were collected using the extinction spectra of the colloidal Au nanozymes for base-line subtraction. The concentrations of oxTMB were calculated on the basis of the measrued absorbance at $652 \mathrm{~nm}$ using the Beer-Lambert Law assuming a molar absorption coefficient of $39,000 \mathrm{M}^{-1} \cdot \mathrm{cm}^{-1} .{ }^{8}$ All kinetic measurements were repeated 3 times under each reaction condition and the standard deviations obtained from the triplicate measuremetns were shown as the error bars in the kinetic resutls.

\section{S1.6 Calculations of Apparent Quantum Efficiency}

The apparent quantum efficiency (AQE) of the photo-enhanced reactions was defined as the ratio between the number of hot electrons utilized to enhance the reactions ( $\left.n_{\text {hot e}}-\right)$ and the number of photons incident on the samples ( $n_{\text {photon }}$ ) over a certain reaction time at the early stage of the reactions. According to the mechanism illustrated in Figure 1A in the main text, one hot electron injected into the surface-adsorbed $\mathrm{H}_{2} \mathrm{O}_{2}$ induces the generation of two reactive hydroxyls on the nanozyme surface, producing two TMB cationic radicals that combine into one molecule of oxTMB charge-transfer complex. $n_{\text {hot }} \mathrm{e}^{-}$is assumed to be equal to the number of photo-generated oxTMB molecules ( $\left.n_{\text {oxTMB }}\right)$. Therefore,

$$
A Q E=\frac{n_{\text {oxTMB }}}{n_{\text {photon }}} \quad \text { (Equation S1). }
$$

$n_{\text {oxTMB }}$ was calculated by subtracting the number of oxTMB produced in dark from the total number of oxTMB produced under laser illumination.

$$
n_{\text {oхTMB }}=\left[v_{0}(\text { laser on })-v_{0}(\text { laser off })\right] \times t \times V \times N_{A} \quad \text { (Equation S2), }
$$

where $v_{0}$ is the initial velocity, $t$ is the reaction time, $V$ is the total volume of the reaction solution (3.0 $\mathrm{mL}$ ), and $N_{\mathrm{A}}$ is the Avogadro's number (6.022 x $10^{23}$ molecules mole $\left.{ }^{-1}\right)$, respectively.

$n_{\text {photon }}$ was calculated using the following equation:

$$
n_{\text {photon }}=\frac{P_{\mathrm{ex}} \times \lambda_{e x} \times t}{h c} \quad \text { (Equation S3), }
$$

where $P_{\text {ex }}$ is the power of the incident laser, $\lambda_{\text {ex }}$ is the laser excitation wavelength, $c$ is the speed of light in air $\left(3.0 \times 10^{8} \mathrm{~m} \mathrm{~s}^{-1}\right)$, and $h$ is the Planck constant $\left(6.626 \times 10^{-34} \mathrm{~m}^{2} \mathrm{~kg} \mathrm{~s}^{-1}\right)$, respectively.

Combining Equations S1, S2, and S3, we obtained the following equation for the calculations of AQE,

$$
A Q E=\frac{h \times c \times V \times N_{A} \times\left[v_{0}(\text { laser on })-v_{0}(\text { laser off })\right]}{P_{\text {ex }} \times \lambda_{e x}} \quad \text { (Equation S4). }
$$

Under the current experimental conditions, more than $90 \%$ of the incident photons were transmitted through the samples because of the low concentrations of the colloidal nanozymes. In addition, a significant fraction of the photons was scattered rather than absorbed by the nanoparticles at the plasmon resonance 
wavelengths due to the relatively large particle sizes. Therefore, the intrinsic quantum efficiency, which was defined as the ratio between the number of hot electrons harnessed for the reactions and the number of photons absorbed by the nanozymes, was estimated to be more than one order of magnitude higher than the as-calculated AQE.

\section{S1.7 Analysis of the Kinetics Results Using the Michaelis-Menten Model}

We analyzed the kinetics of the nanozyme-catalyzed reactions both in dark and under laser illumination using the Michaelis-Menten model, ${ }^{9}$ which relates $v_{0}$ to the initial concentrations of the substrates, $C$, through the following equation:

$$
v_{0}=\frac{v_{\max } \times C}{\left(K_{m}+C\right)} \quad \text { (Equation S5), }
$$

where $K_{\mathrm{m}}$ is the Michaelis constant, and $v_{\max }$ represents the maximal $v_{0}$ at saturating $C$. In a typical enzymatic reaction obeying the Michaelis-Menten kinetics, a substrate $(S)$ binds reversibly to an enzyme $(E)$ to form an enzyme-substrate complex $(E S)$ before transforming irreversibly into the product $(P)$, which can be schematically represented as follows:

$$
E+S \stackrel{k_{\text {on }}}{\underset{k_{\text {off }}}{\rightleftharpoons}} E S \stackrel{k_{\text {cat }}}{\longrightarrow} P+E
$$

(Equation S6).

Here, $k_{o n}, k_{o f f}$, and $k_{c a t}$ are the rate constants for the ES association, ES dissociation, and product formation, respectively. $k_{c a t}$ and $\left(k_{c a t} / K_{\mathrm{m}}\right)$ are also known as the turnover number and the catalytic efficiency of the enzyme, respectively. $K_{\mathrm{m}}$ is related to $k_{o n}, k_{o f f}$, and $k_{c a t}$ through the following equation:

$$
K_{\mathrm{m}}=\frac{k_{\text {off }}+k_{\mathrm{cat}}}{k_{\text {on }}} \quad \text { (Equation S7). }
$$

At saturating substrate concentration, $v_{0}$ reaches $v_{\max }$ and the concentration of $E S$ becomes equivalent to the total concentration of the enzyme, $[E]_{0}$. Therefore,

$$
v_{\max }=k_{c a t}[E]_{0} \quad \text { (Equation } \mathrm{S} 8 \text { ). }
$$

For an enzymatic reaction obeying the Michaelis-Menten kinetics, the reciprocal of $v_{0}$ varies linearly with the reciprocal of the substrate concentration, and the values of $K_{\mathrm{m}}$ and $v_{\max }$ were obtained by fitting the experimental results with the Lineweaver-Burk equation:

$$
\frac{1}{v_{0}}=\frac{K_{m}}{v_{\max }} \times \frac{1}{C}+\frac{1}{v_{\max }} \quad \text { (Equation S9), }
$$

in which the intercept and the slope correspond to $\left(1 / v_{\max }\right)$ and $\left(K_{\mathrm{m}} / v_{\max }\right)$, respectively.

\section{S1.8 Synthesis of Au SRNP@Polydopamine Core-Shell Nanoparticles and Surface-Enhanced Raman Scattering (SERS) of Thionine}

The surfaces of the Au SRNPs were further coated with a thin polymer layer composed of polydopamine (PDA) through seed-mediated self-polymerization of dopamine. ${ }^{10}$ Briefly, $1 \mathrm{~mL}$ of the $\mathrm{Au}$ SRNPs $\left(4.8 \times 10^{9}\right.$ particles $\left.\mathrm{mL}^{-1}\right)$ was centrifuged at $5000 \mathrm{rpm}$ for $10 \mathrm{~min}$, followed by redispersion in $5 \mathrm{~mL}$ of Tirs-base aqueous solution $(10 \mathrm{mM}, \mathrm{pH}=8.5)$. Then $2 \mathrm{~mL}$ of iso-propanol and $0.3 \mathrm{~mL}$ of 
dopamine hydrochloride aqueous solution $\left(2 \mathrm{mg} \mathrm{mL}^{-1}\right)$ were added. The reactant mixtures were kept under magnetic stir at $400 \mathrm{rpm}$ for $20 \mathrm{~h}$ at room temperature. Finally, the suspension was centrifuged at $4000 \mathrm{rpm}$ for $10 \mathrm{~min}$, washed with water twice, and finally dispersed in water for further use.

As shown by the TEM images in Figures S18A and 18B, the thickness of the PDA shells could be controlled in the sub-10 nm regime. The coating of Au SRNPs (Au-785) with a sub-10 nm thick PDA shell caused a spectral red-shift of the plasmon peak by $\sim 15 \mathrm{~nm}$ due to increased local refractive index surrounding the Au SRNPs (Figure S18C). To prepare the samples for SERS measurements, colloidal Au-785 and Au-785@PDA particles were incubated with $5 \mu \mathrm{M}$ thionine aqueous solution for $1 \mathrm{~h}$ at room temperature, and then centrifuged and redispersed in $\mathrm{H}_{2} \mathrm{O}$. The concentrations of thionine-coated Au-785 and Au-785@PDA particles were both kept at $2.0 \times 10^{9}$ particles $\mathrm{mL}^{-1}$ for SERS measurements. SERS spectra were collected using a Bayspec Nomadic confocal Raman microscope built on an Olympus BX51 reflected optical system with a $785 \mathrm{~nm}$ continuous wave excitation laser. The excitation laser was focused on colloidal samples with a 10x objective [Numerical Aperture (NA) $=0.30$, working distance $(\mathrm{WD})=11.0 \mathrm{~mm}$, Olympus MPLFLN, excitation volume of $\sim 100 \mathrm{fL}]$. The laser power for Raman excitation was $5.0 \mathrm{~mW}$. The spectral acquisition time was $5 \mathrm{~s}$. As shown in Figure S18D, the SERS signals of thionine adsorbed on Au-785@PDA core-shell nanoparticles were only slightly lower than those of thionine adsorbed on the surfaces of Au-785, suggesting strong local-field enhancements on the outer surfaces of Au-785@PDA core-shell nanoparticles. However, the Au-785@PDA core-shell nanoparticles exhibited diminished peroxidase-mimicking activities both in dark and upon plasmonic excitations (Figure S18E), strongly indicating that the catalytically active sites were essentially on the $\mathrm{Au}$ surfaces and the thin polymer layer effectively blocked the ejection of hot electrons from the $\mathrm{Au}$ nanoparticles to the molecular adsorbates. These observations provided additional evidence that the plasmon-enhanced peroxidase-mimicking activities of Au SRNPs stemmed essentially from hot electrons rather than local field enhancements, though the abundance of hot electrons at the hot spots was intimately tied to the local field enhancements.

\section{S2. Tables}

Table S1. Details for the preparation of $100 \mathrm{~mL} 10 \mathrm{x}$ HAc-NaAc buffers with different $\mathrm{pH}$ values. When used, these buffers were diluted by 10 times.

\begin{tabular}{|c|c|c|}
\hline $\mathbf{p H}$ & Mass of HAc $/ \mathbf{g}$ & Mass of $\mathbf{N a A c} / \mathbf{g}$ \\
\hline 3.0 & 0.5900 & 0.02380 \\
\hline 4.0 & 0.5098 & 0.2055 \\
\hline 4.5 & 0.3844 & 0.4898 \\
\hline 5.0 & 0.2161 & 0.8710 \\
\hline 6.0 & 0.03200 & 1.288 \\
\hline 7.0 & 0.003400 & 1.353 \\
\hline 8.0 & 0.0003000 & 1.360 \\
\hline
\end{tabular}




\section{S3. Additional Figures}
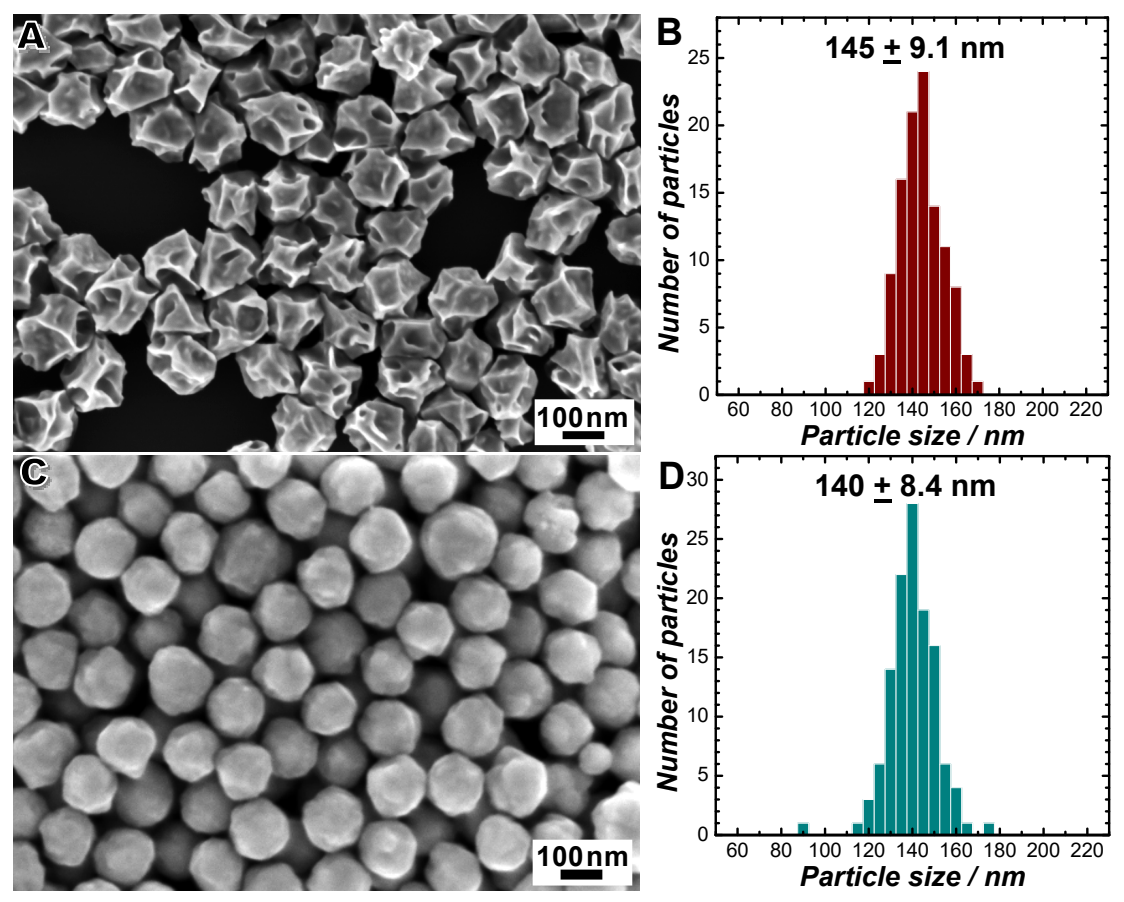

Figure S1. SEM images of (A) Au SRNPs and (C) Au QSNPs. Size distributions of (B) Au SRNPs and (D) Au QSNPs.

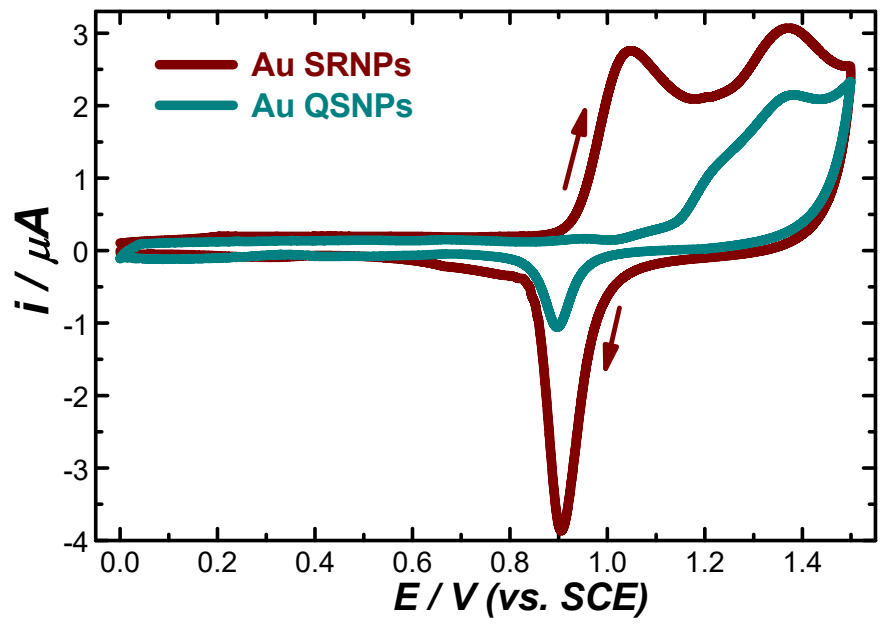

Figure S2. Cyclic voltammetry curves of Au SRNPs $(145 \pm 9.1 \mathrm{~nm}$ in diameter) and QSNPs $(140 \pm 8.4 \mathrm{~nm}$ in diameter) on GCEs in $0.5 \mathrm{M} \mathrm{H}_{2} \mathrm{SO}_{4}$ at a potential sweep rate of $5.0 \mathrm{mV} \mathrm{s}^{-1}$. The arrows indicate the potential sweep directions. On each GCE, there were nominally $3 \times 10^{7}$ nanoparticles $(5.0 \mu \mathrm{L}$ of $9.6 \mathrm{pM}$ colloidal QSNPs or SRNPs dried on each GCE). 

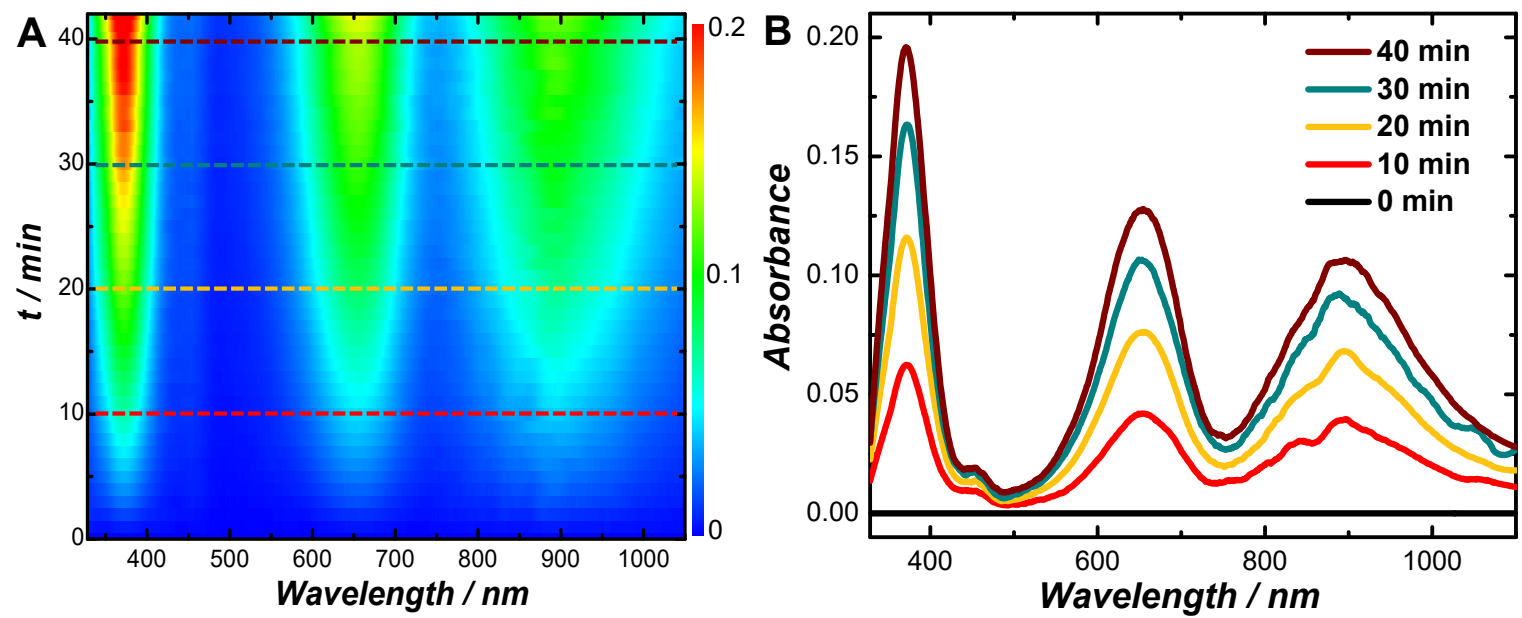

Figure S3. (A) Temporal evolution of UV-visible-near infrared absorption spectra during TMB oxidation catalyzed by Au SRNPs (Au-785) in dark. (B) Snapshot absorption spectra recorded at reaction times of 0 , $10,20,30$, and $40 \mathrm{~min}$, as labeled by the horizontal dash lines in panel A. The temperature of the bulk solution was at $25{ }^{\circ} \mathrm{C}$. The $\mathrm{pH}$ of the reaction solutions was 4.5. The initial concentrations of the $\mathrm{Au}$ nanozymes, $\mathrm{H}_{2} \mathrm{O}_{2}$, and TMB were $180 \mathrm{fM}, 568 \mathrm{mM}$, and $750 \mu \mathrm{M}$, respectively.

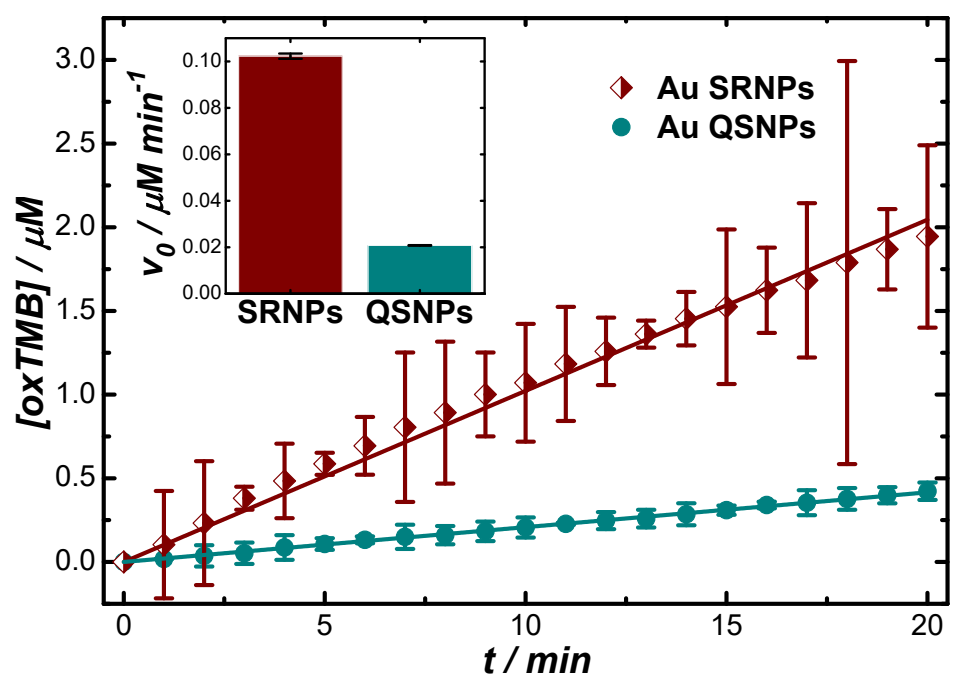

Figure S4. Comparison of catalytic activities of Au SRNPs (Au-785) and QSNPs in dark. The values of $v_{0}$ were obtained by fitting the linear part of the kinetic curves at the early stage of the reactions $(0-20 \mathrm{~min})$ and were compared in the inset. The error bars represent the standard deviations of 3 experimental runs under each condition. The initial concentrations of $\mathrm{H}_{2} \mathrm{O}_{2}$ and $\mathrm{TMB}$ were $568 \mathrm{mM}$ and $750 \mu \mathrm{M}$, respectively. The nanozyme concentrations were $180 \mathrm{fM}$ for SRPNs and $750 \mathrm{fM}$ for QSNPs, respectively, such that the overall surface areas of SRNPs and QSNPs were nominally the same when comparing their catalytic activities. The $\mathrm{pH}$ was fixed at 4.5 and the temperature of the bulk solution was at $25^{\circ} \mathrm{C}$. 


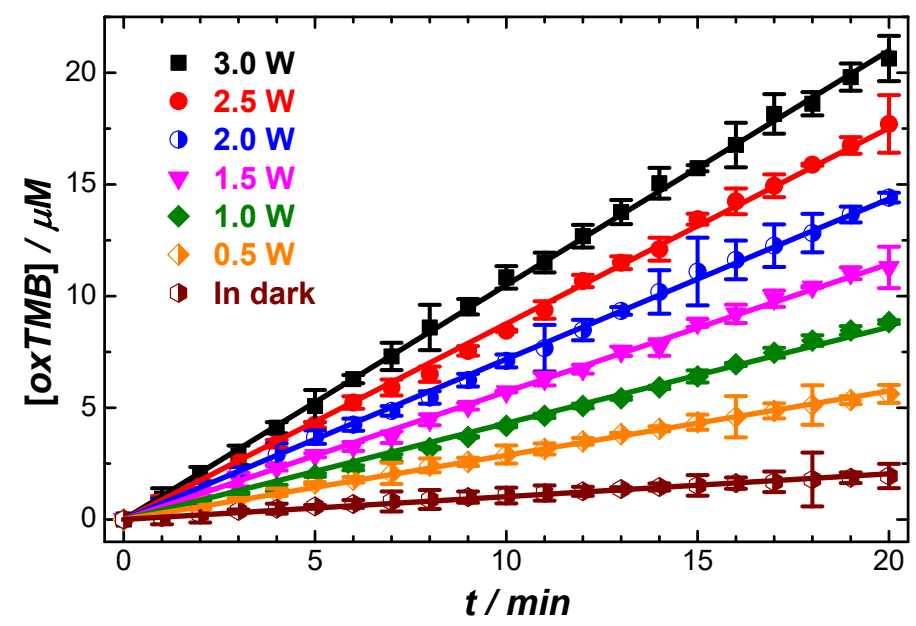

Figure S5. Temporal evolution of oxTMB concentrations at the early stage of the reactions $(0-20 \mathrm{~min})$ catalyzed by $\mathrm{Au}$ SRNPs (Au-785) under $785 \mathrm{~nm}$ laser illumination at various $P_{\mathrm{ex}}$ in the range of 0-3.0 W. The error bars represent the standard deviations of 3 experimental runs under each condition. The least squares fitting results were shown as solid lines. The temperature of the bulk solutions was maintained at 25 $\pm 1{ }^{\circ} \mathrm{C}$ using a circulating water bath, and the $\mathrm{pH}$ of the reaction solutions was kept at 4.5. The initial concentrations of the $\mathrm{Au}$ nanozymes, $\mathrm{H}_{2} \mathrm{O}_{2}$, and $\mathrm{TMB}$ were $180 \mathrm{fM}, 568 \mathrm{mM}$, and $750 \mu \mathrm{M}$, respectively, for all the kinetic measurements.

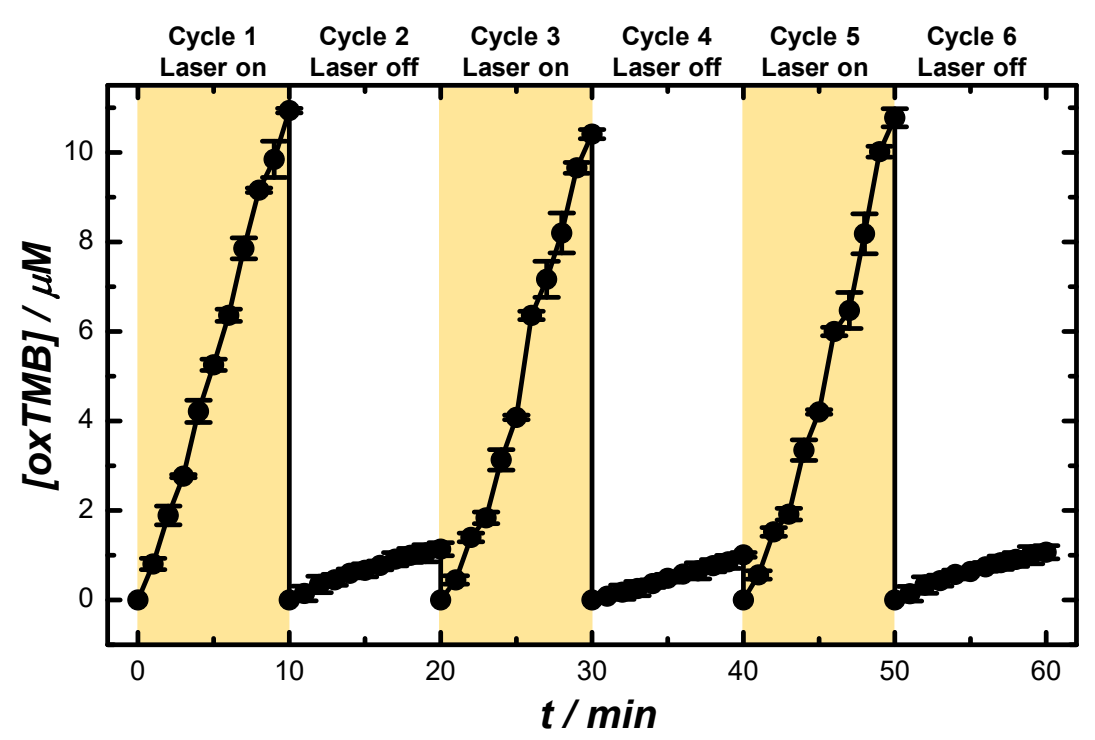

Figure S6. Temporal evolution of oxTMB concentrations during the reactions catalyzed by Au SRNPs (Au$785)$ over multiple cycles of laser illumination $\left(\lambda_{\mathrm{ex}}=785 \mathrm{~nm}, P_{\mathrm{ex}}=3.0 \mathrm{~W}\right)$ and dark reactions. The Au nanozymes were recycled through centrifugation after each reaction cycle and then remixed with TMB for the next reaction cycle. The temperature of the bulk solutions was maintained at $25 \pm 1{ }^{\circ} \mathrm{C}$ using a circulating water bath, and the $\mathrm{pH}$ of the reaction solutions was kept at 4.5. The initial concentrations of the $\mathrm{Au}$ nanozymes, $\mathrm{H}_{2} \mathrm{O}_{2}$, and TMB were $180 \mathrm{fM}, 568 \mathrm{mM}$, and $750 \mu \mathrm{M}$, respectively. The error bars represent the standard deviations of 3 experimental runs. 

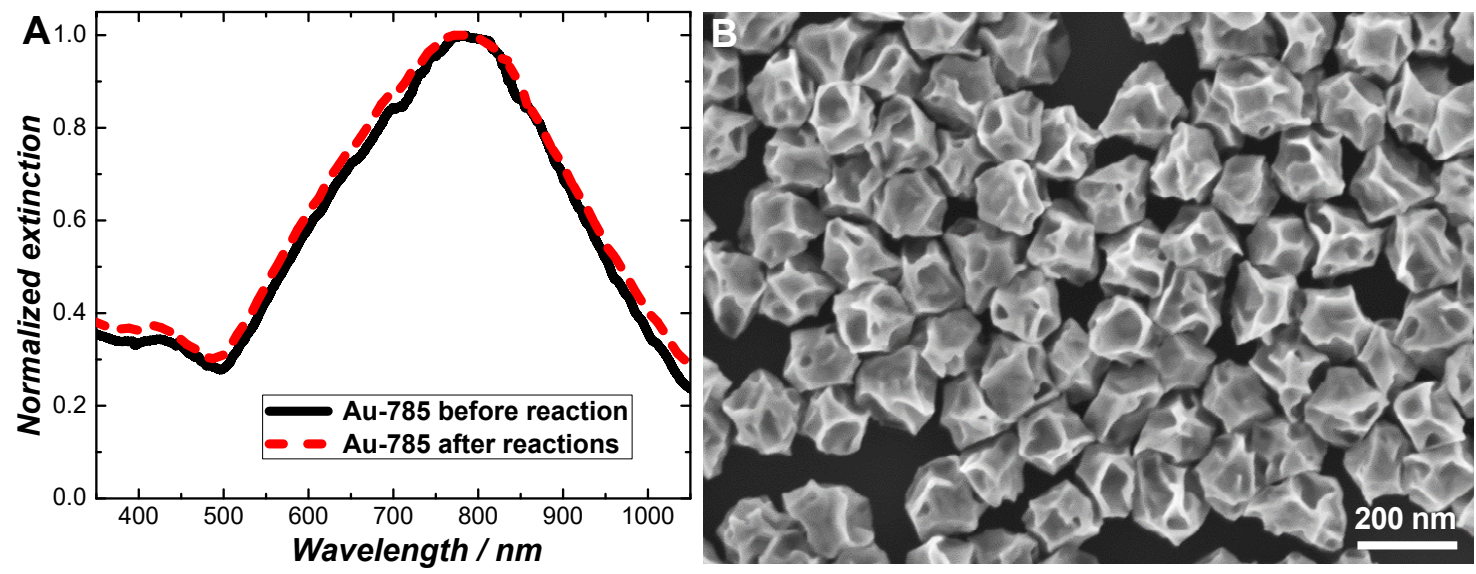

Figure S7. Extinction spectra of Au SRNPs (Au-785) before reactions and after 3 cycles of catalytic reactions under laser illumination and in dark. The Au nanozymes were recycled through centrifugation after 3 cycles of laser illumination $\left(\lambda_{\mathrm{ex}}=785 \mathrm{~nm}, P_{\mathrm{ex}}=3.0 \mathrm{~W}\right)$ and dark reactions (see Figure S6). The temperature of the bulk solutions was maintained at $25 \pm 1{ }^{\circ} \mathrm{C}$ using a circulating water bath, and the $\mathrm{pH}$ of the reaction solutions was kept at 4.5. The initial concentrations of the Au nanozymes, $\mathrm{H}_{2} \mathrm{O}_{2}$, and TMB were $180 \mathrm{fM}, 568$ $\mathrm{mM}$, and $750 \mu \mathrm{M}$, respectively.
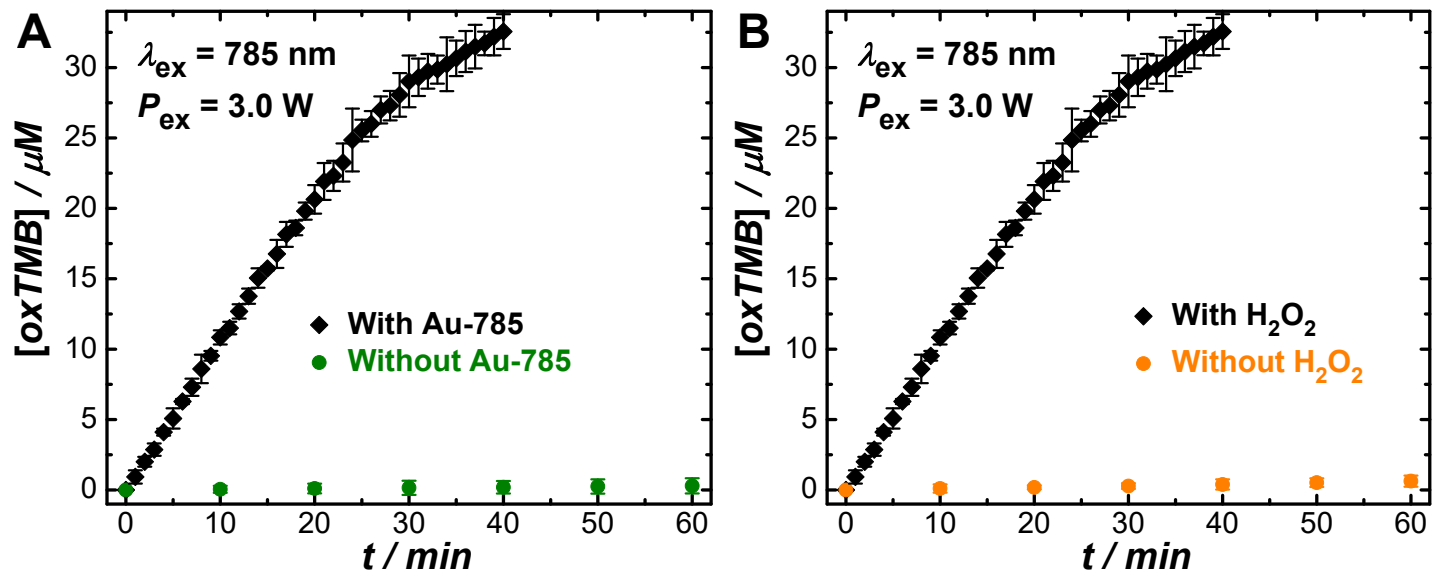

Figure S8. (A) Temporal evolution of oxTMB concentrations in the presence of $\mathrm{Au}-785$ (180 $\mathrm{fM}$ ) and in the absence of Au-785 at $\lambda_{\text {ex }}$ of $785 \mathrm{~nm}$ and $P_{\text {ex }}$ of $3.0 \mathrm{~W}$. The initial concentrations of $\mathrm{H}_{2} \mathrm{O}_{2}$ and TMB were 568 $\mathrm{mM}$ and $750 \mu \mathrm{M}$, respectively. (B) Temporal evolution of oxTMB concentrations catalyzed by Au-785 in the presence of $\mathrm{H}_{2} \mathrm{O}_{2}(568 \mathrm{mM})$ and in the absence of $\mathrm{H}_{2} \mathrm{O}_{2}$ at $\lambda_{\text {ex }}$ of $785 \mathrm{~nm}$ and $P_{\text {ex }}$ of $3.0 \mathrm{~W}$. The initial concentration of TMB was $750 \mu \mathrm{M}$. The concentration of Au-785 was $180 \mathrm{fM}$. In all these experiments, the temperature of the bulk solutions was maintained at $25 \pm 1{ }^{\circ} \mathrm{C}$ using a circulating water bath, and the $\mathrm{pH}$ of the reaction solutions was kept at 4.5. The error bars represent the standard deviations of 3 experimental runs under each condition. 

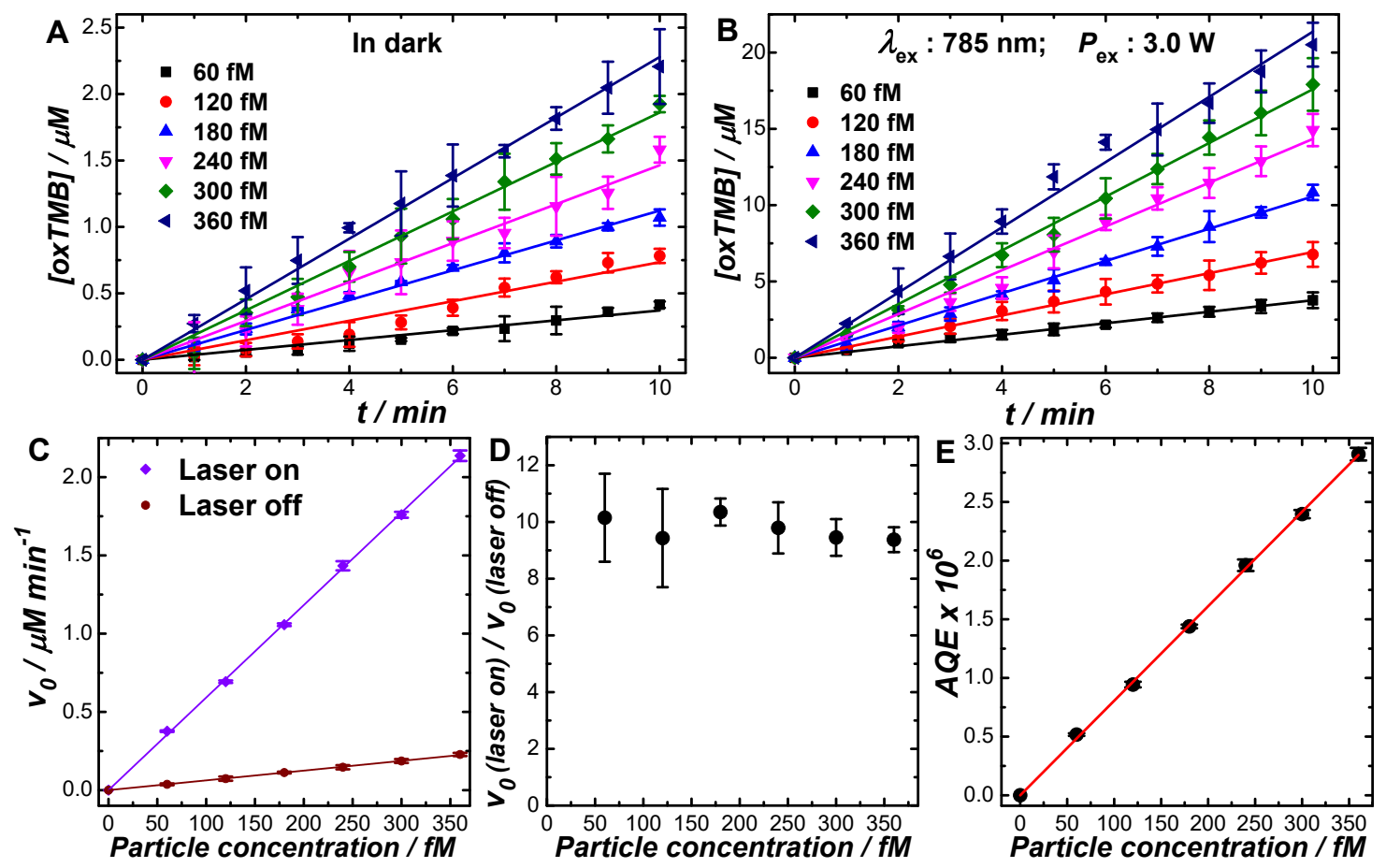

Figure S9. Temporal evolution of oxTMB concentrations at the early stage of the reactions catalyzed by various concentrations of Au-785 nanozymes (A) in dark and (B) under laser illumination $\left(\lambda_{\mathrm{ex}}=785 \mathrm{~nm}, P_{\mathrm{ex}}\right.$ $=3.0 \mathrm{~W})$. The error bars represent the standard deviations of 3 experimetnal runs under each condition. The least squares fitting results were shown as solid lines. (C) Plots of $v_{0} v s$. nanozyme concentration for the reactions in dark and under laser illumination $\left(\lambda_{\mathrm{ex}}=785 \mathrm{~nm}, P_{\mathrm{ex}}=3.0 \mathrm{~W}\right)$. (D) Enhancements of $v_{0}$ caused by laser illumination at various nanozyme concentrations. (E) Plots of $A Q E v s$. nanozyme concentration. The temperature of the bulk solutions was controlled at $25 \pm 1{ }^{\circ} \mathrm{C}$ using a water bath, and the $\mathrm{pH}$ of the reaction solutions was kept at 4.5. The initial concentrations of $\mathrm{H}_{2} \mathrm{O}_{2}$ and $\mathrm{TMB}$ were 568 and $750 \mu \mathrm{M}$, respectively.
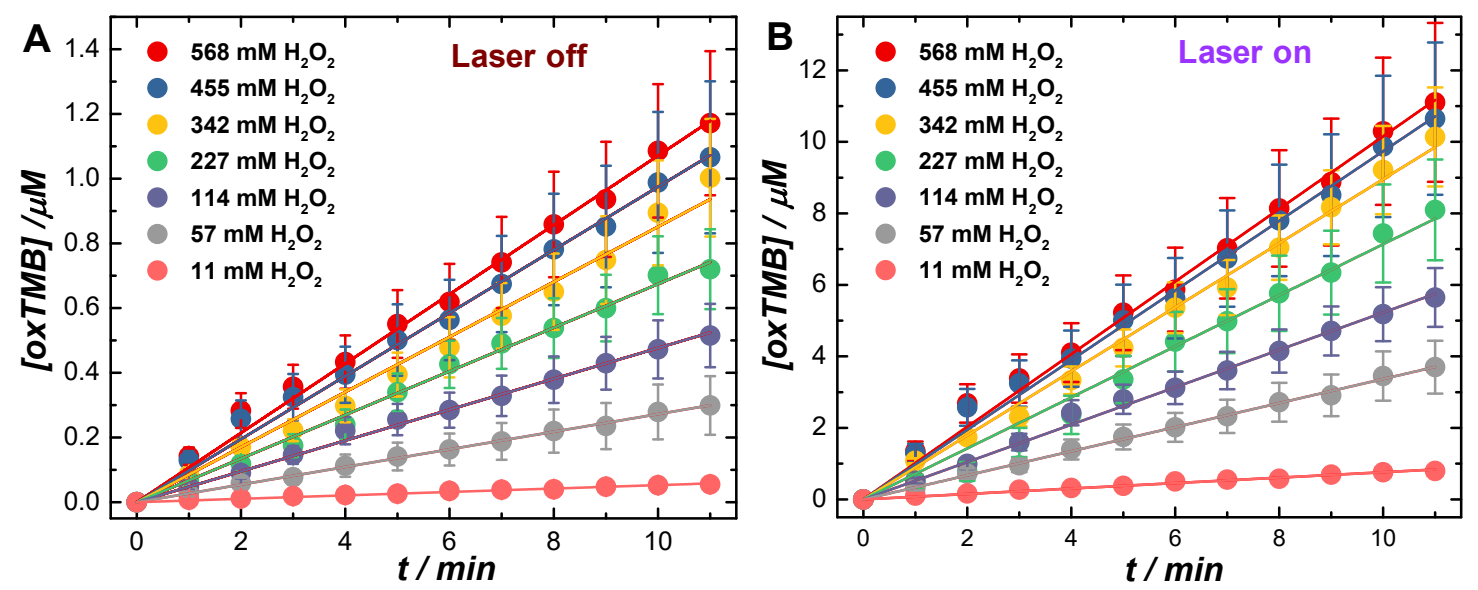

Figure S10. Temporal evolution of oxTMB concentrations at the early stage of the reactions catalyzed by Au-785 nanozymes (A) in dark and (B) under laser illumination $\left(\lambda \lambda_{\mathrm{ex}}=785 \mathrm{~nm}, P_{\mathrm{ex}}=3.0 \mathrm{~W}\right)$ at various $\mathrm{H}_{2} \mathrm{O}_{2}$ concentrations. The temperature of the bulk solutions was controlled at $25 \pm 1{ }^{\circ} \mathrm{C}$ using a water bath, and the $\mathrm{pH}$ of the reaction solutions was kept at 4.5. The initial concentrations of the Au nanozymes and TMB were kept at $180 \mathrm{fM}$ and $750 \mu \mathrm{M}$, respectively. 

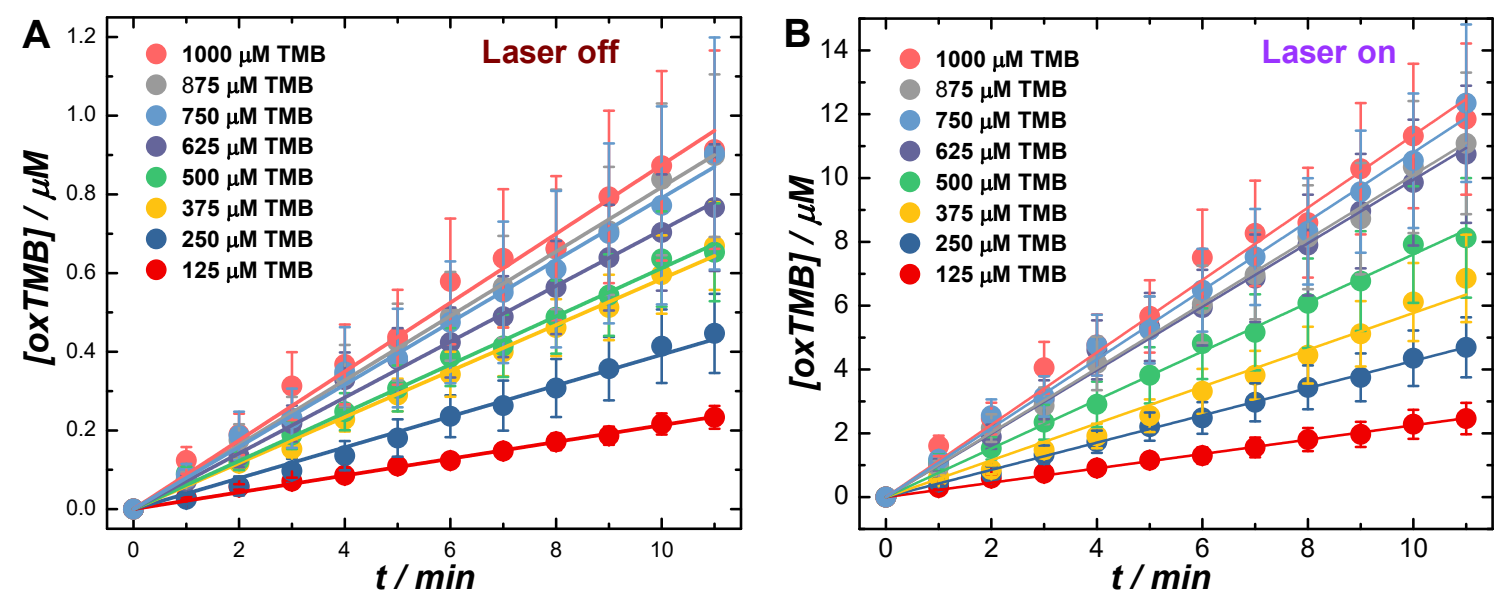

Figure S11. Temporal evolution of oxTMB concentrations at the early stage of the reactions catalyzed by Au-785 nanozymes (A) in dark and (B) under laser illumination $\left(\lambda_{\mathrm{ex}}=785 \mathrm{~nm}, P_{\mathrm{ex}}=3.0 \mathrm{~W}\right)$ at various TMB concentrations. The temperature of the bulk solutions was controlled at $25 \pm 1{ }^{\circ} \mathrm{C}$ using a water bath, and the $\mathrm{pH}$ of the reaction solutions was kept at 4.5. The initial concentrations of the Au nanozymes and $\mathrm{H}_{2} \mathrm{O}_{2}$ were kept at $180 \mathrm{fM}$ and $568 \mathrm{mM}$, respectively.
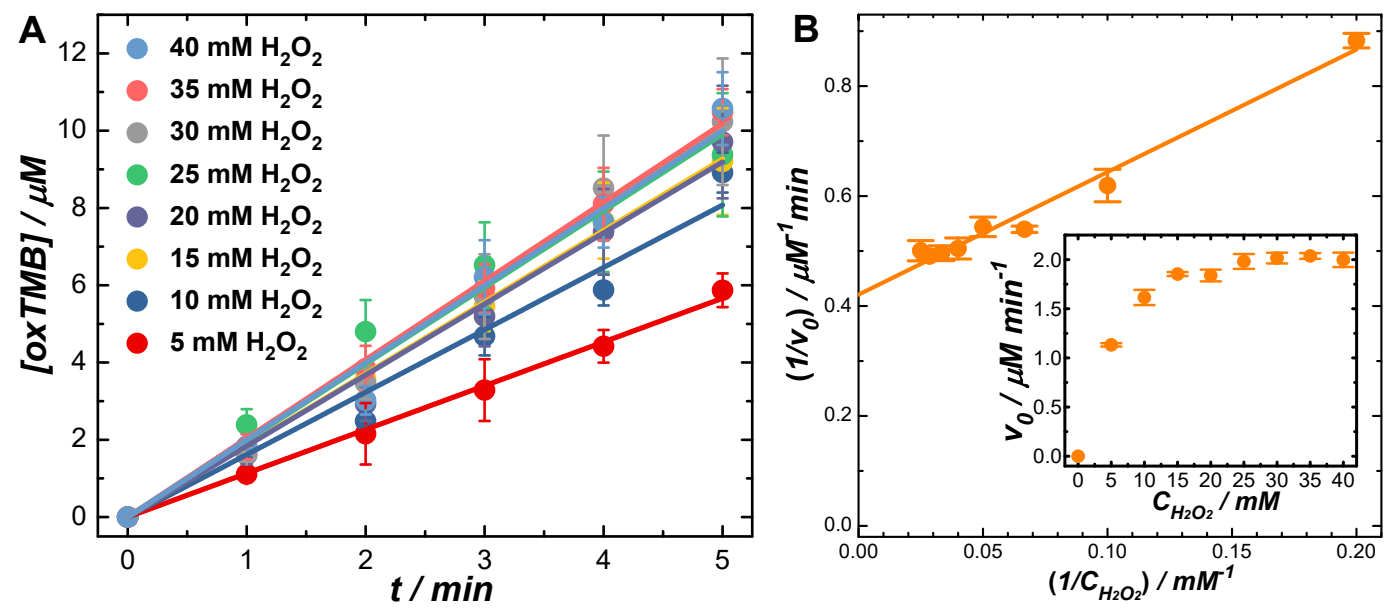

Figure S12. (A) Temporal evolution of oxTMB concentrations at the early stage of HRP-catalyzed TMB oxidation at various $\mathrm{H}_{2} \mathrm{O}_{2}$ concentrations. The initial concentrations of HRP and TMB were $11 \mathrm{pM}$ and 800 $\mu \mathrm{M}$, respectively. The $\mathrm{pH}$ was fixed at 4.5 and the temperature was controlled at $25{ }^{\circ} \mathrm{C}$. The error bars of the data points represent the standard deviations of 3 experimetnal runs under each condition. The least squares fitting results were shown as solid lines. (B) The Lineweaver-Burk plots revealing the Michaelis-Menten reaction kinetics. The inset shows the plots of $v_{0} v s . \mathrm{H}_{2} \mathrm{O}_{2}$ concentration. 

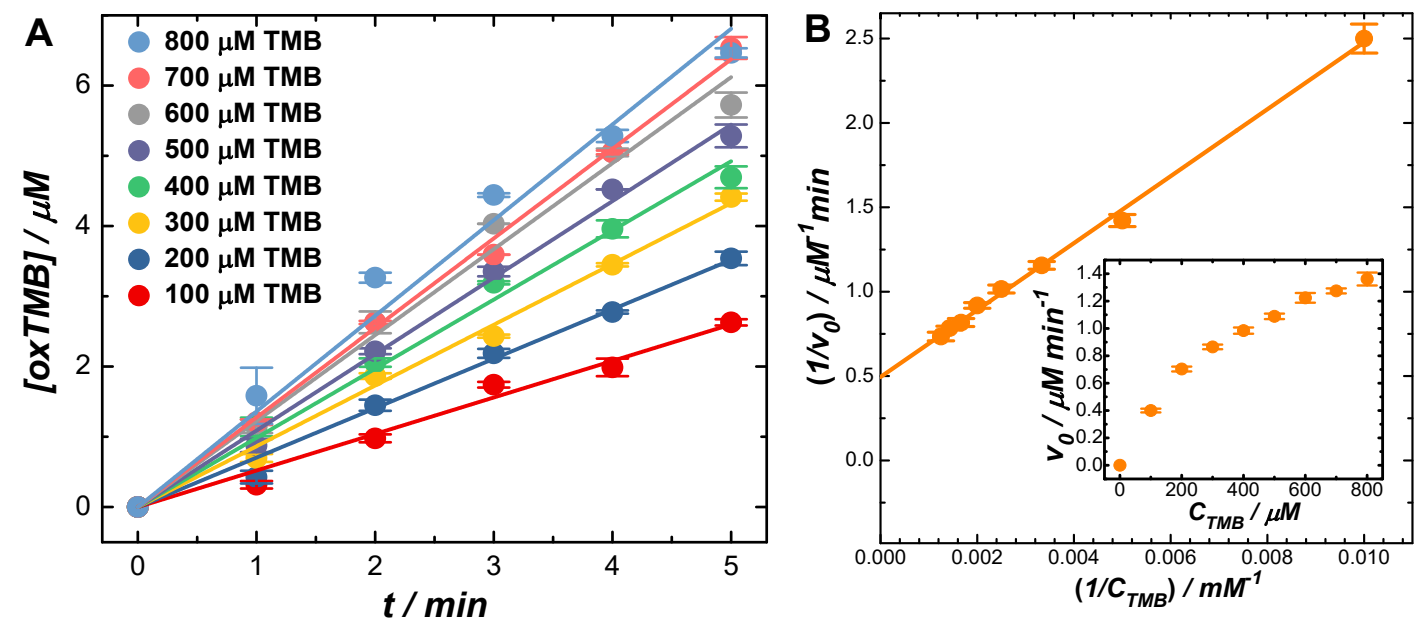

Figure S13. (A) Temporal evolution of oxTMB concentrations at the early stage of HRP-catalyzed TMB oxidation at various TMB concentrations. The initial concentrations of $\mathrm{HRP}$ and $\mathrm{H}_{2} \mathrm{O}_{2}$ were $11 \mathrm{pM}$ and 568 $\mathrm{mM}$, respectively. The $\mathrm{pH}$ was fixed at 4.5 and the temperature was controlled at $25^{\circ} \mathrm{C}$. The error bars of the data points represent the standard deviations of 3 experimetnal runs under each condition. The least squares fitting results were shown as solid lines. (B) The Lineweaver-Burk plots revealing the Michaelis-Menten reaction kinetics. The inset shows the plots of $v_{0} v s$. TMB concentration.
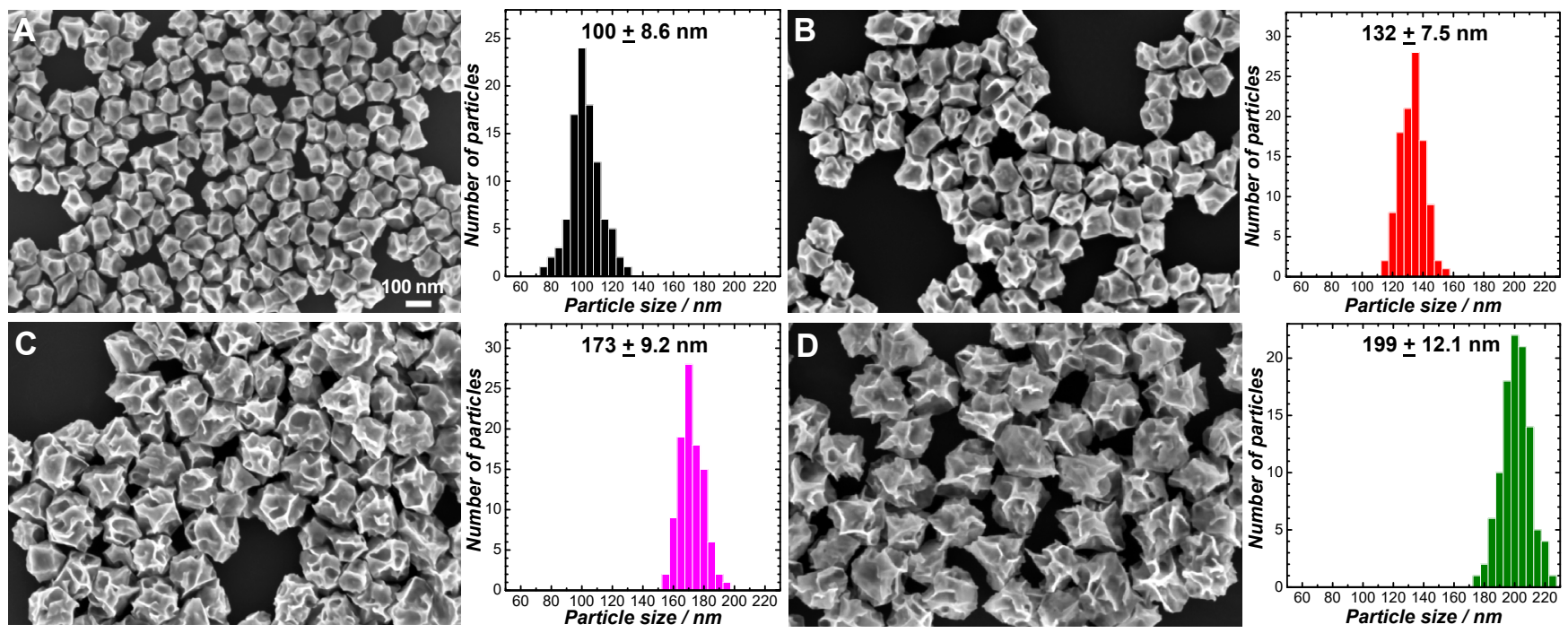

Figure S14. SEM pictures (left panels) and size-distributions (right panels) of Au SRNPs with various average particle sizes. The distributions of particle sizes were obtained based on the SEM images of each sample using the Image J software. The four samples were labeled as (A) Au-638, (B) Au-714, (C) Au-858, and (D) Au-980, respectively, according to their plasmon resonance peak positions in the extinction spectra (see Figure 3A). All SEM images share the same scale bar in panel A. 


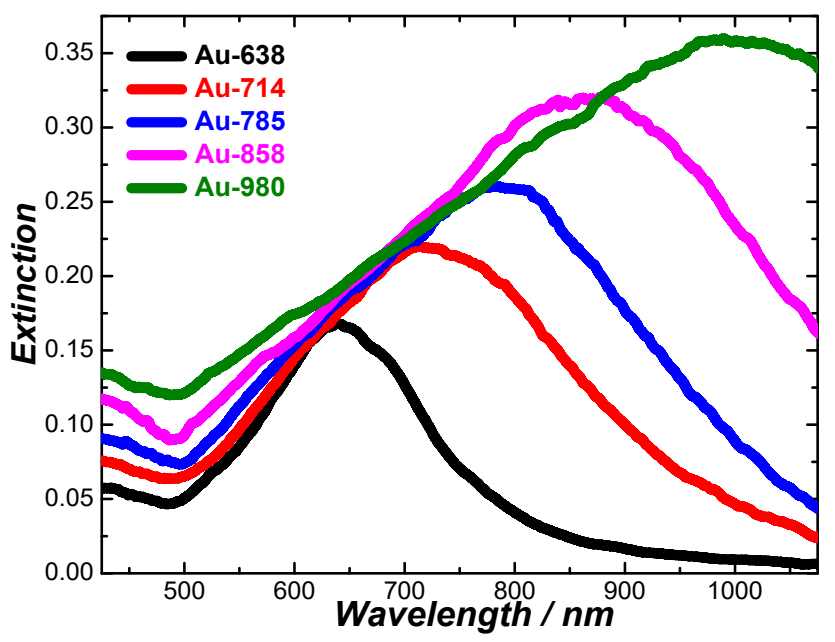

Figure S15. Extinction spectra of colloidal suspensions of various Au SRNP samples at nominally the same particle concentration of $2 \mathrm{pM}$.
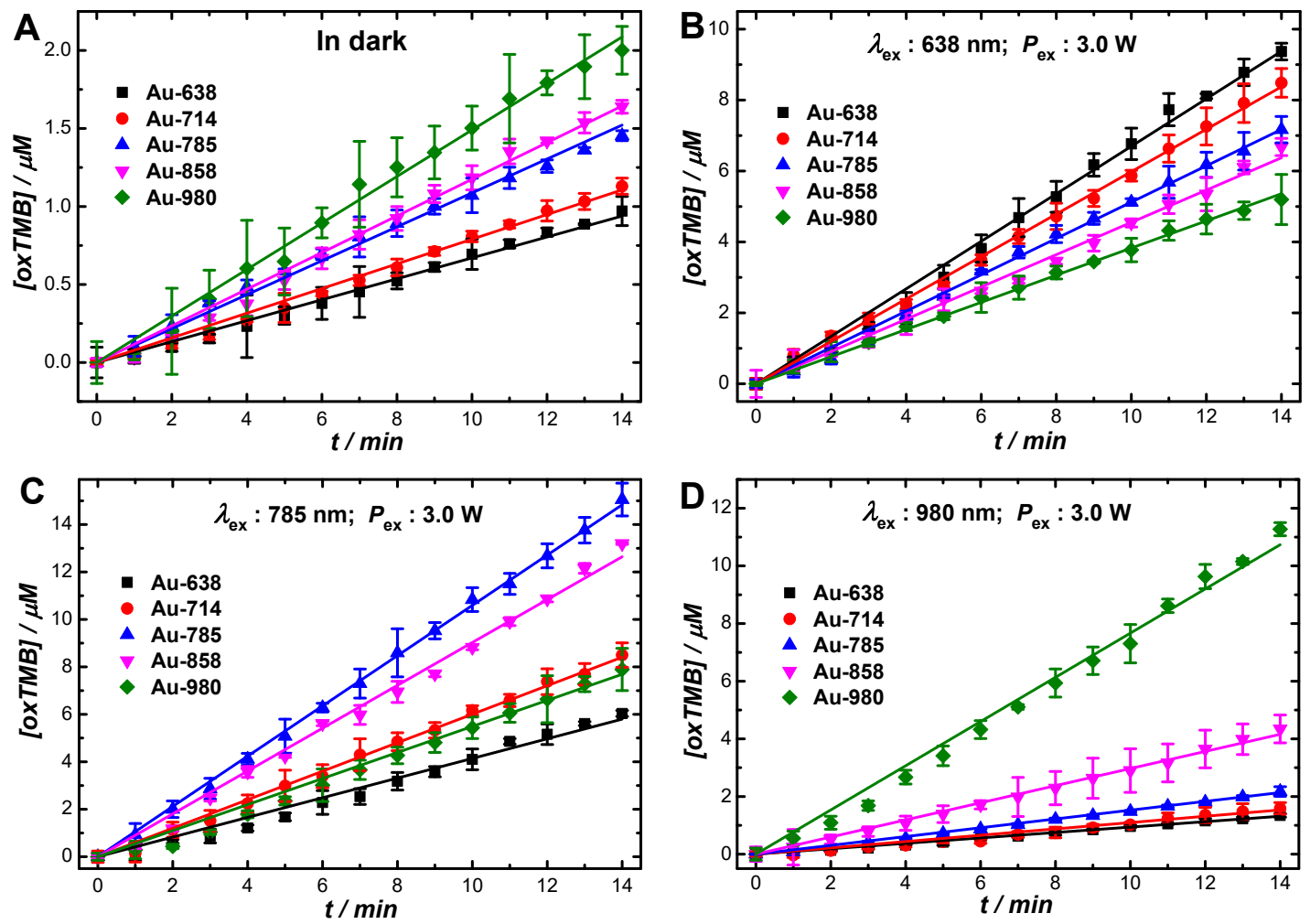

Figure S16. Temporal evolution of oxTMB concentrations at the early stage of the reactions catalyzed by various Au nanozymes (A) in dark and under laser illumination: (B) $\lambda_{\mathrm{ex}}=638 \mathrm{~nm}, P_{\mathrm{ex}}=3.0 \mathrm{~W}$; (C) $\lambda_{\mathrm{ex}}=785$ $\mathrm{nm}, P_{\mathrm{ex}}=3.0 \mathrm{~W}$; (D) $\lambda_{\mathrm{ex}}=980 \mathrm{~nm}, P_{\mathrm{ex}}=3.0 \mathrm{~W}$. The temperature of the bulk solutions was controlled at $25 \pm$ $1{ }^{\circ} \mathrm{C}$ using a water bath, and the $\mathrm{pH}$ of the reaction solutions was kept at 4.5. The initial concentrations of the Au nanozymes, $\mathrm{H}_{2} \mathrm{O}_{2}$, and TMB were $180 \mathrm{fM}, 568 \mathrm{mM}$, and $750 \mu \mathrm{M}$, respectively. 

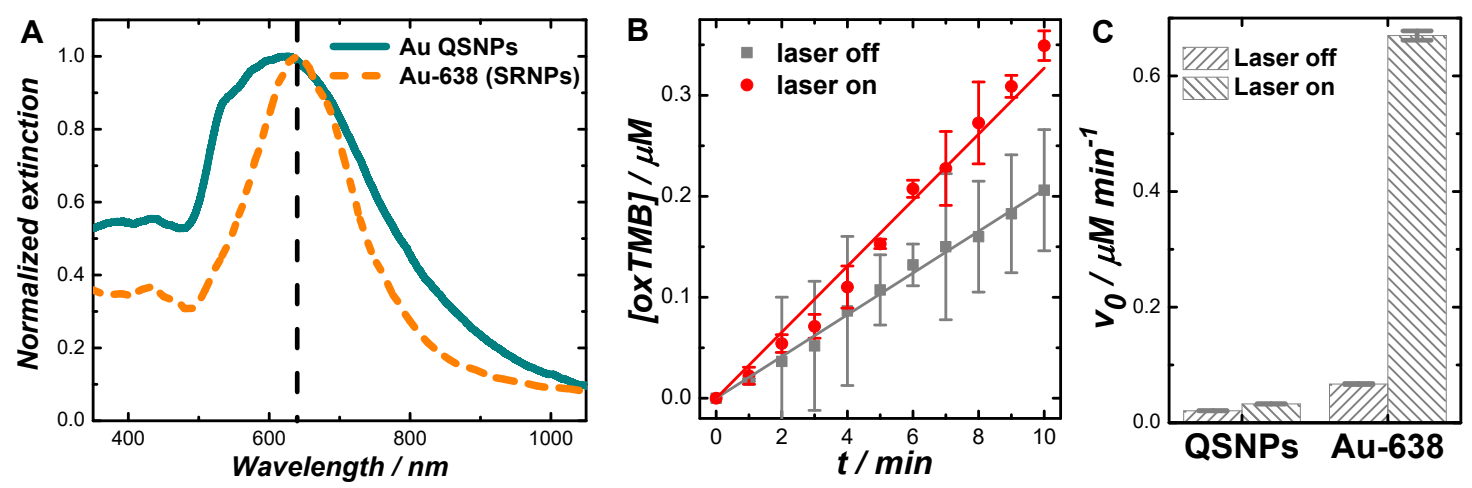

Figure S17. (A) Optical extinction spectra of colloidal Au QSNPs and Au SRNPs (Au-638) with plasmon resonance peaks around $638 \mathrm{~nm}$. The vertical dash line indicates the wavelength of the excitation laser used for photo-enhanced reactions. (B) Comparison of the kinetics of the reactions catalyzed by colloidal Au QSNPs in dark and under laser illumination $\left(\lambda_{\mathrm{ex}}=638 \mathrm{~nm}, P_{\mathrm{ex}}=3.0 \mathrm{~W}\right)$. (C) Comparison of the $v_{0}$ values of $\mathrm{Au}$ QSNPs and Au SRNPs (Au-638) in dark and under laser illumination $\left(\lambda_{\mathrm{ex}}=638 \mathrm{~nm}, P_{\mathrm{ex}}=3.0 \mathrm{~W}\right)$. The nanozyme concentrations were kept at $180 \mathrm{fM}$ for Au-638 (Au SRPNs) and $750 \mathrm{fM}$ for QSNPs, respectively. The initial concentrations of $\mathrm{H}_{2} \mathrm{O}_{2}$ and TMB were $568 \mathrm{mM}$ and $750 \mu \mathrm{M}$, respectively. The $\mathrm{pH}$ was fixed at 4.5 and the temperature of the bulk solutions was controlled at $25 \pm 1{ }^{\circ} \mathrm{C}$.
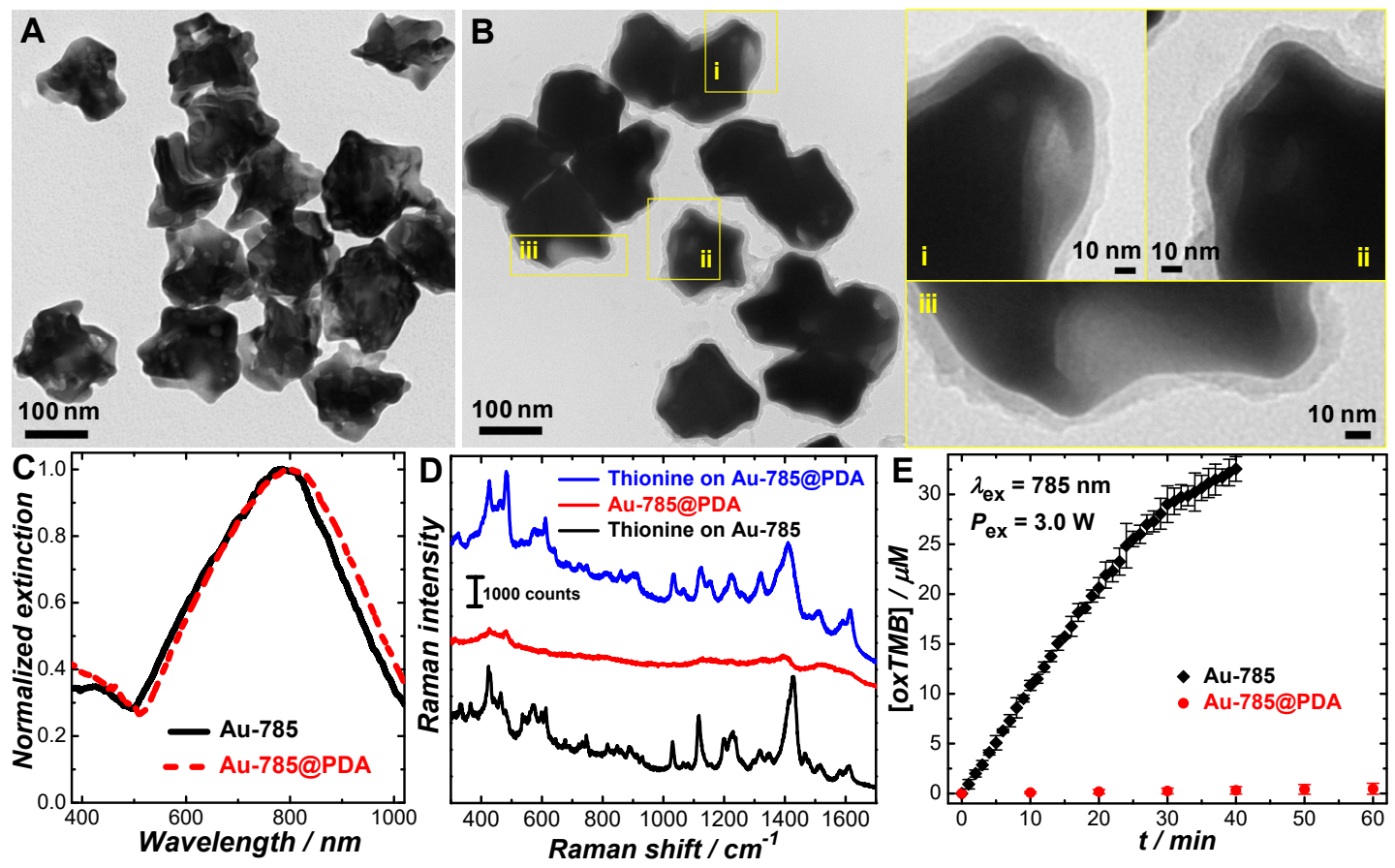

Figure S18. TEM images of (A) Au SRNPs (Au-785) and (B) Au-785@PDA core-shell nanoparticles. Panels i, ii, and iii on the right side of panel B show the high-magnification TEM images highlighting the regions i, ii, and iii in panel B, respectively. (C) Extinction spectra of colloidal Au-785 and Au-785@PDA. (D) SERS spectra collected from Au-785@PDA, thionine-coated Au-785, and thionine-coated Au785@PDA. (E) Temporal evolution of oxTMB concentrations during the reactions catalyzed by Au-785 and Au-785@PDA under laser illumination $\left(\lambda_{\mathrm{ex}}=785 \mathrm{~nm}, P_{\mathrm{ex}}=3.0 \mathrm{~W}\right)$ at $\mathrm{pH}$ of 4.5 . The temperature of the bulk solutions was controlled at $25 \pm 1{ }^{\circ} \mathrm{C}$ using a water bath. The concentrations of the Au-785 and Au@PDA were both kept at $180 \mathrm{fM}$. The concentrations of $\mathrm{H}_{2} \mathrm{O}_{2}$ and TMB were $568 \mathrm{mM}$ and $750 \mu \mathrm{M}$, respectively. 

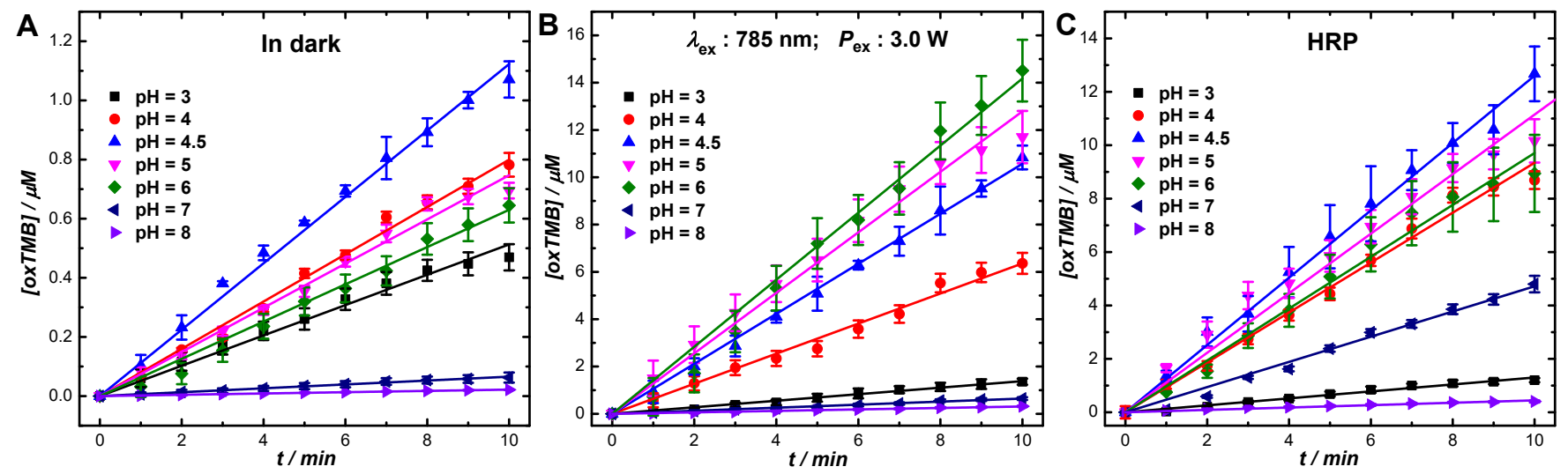

Figure S19. Temporal evolution of oxTMB concentrations at the early stage of the reactions catalyzed by Au-785 (A) in dark and (B) under laser illumination ( $\lambda_{\mathrm{ex}}=785 \mathrm{~nm}, P_{\mathrm{ex}}=3.0 \mathrm{~W}$ ) at various pHs. (C) Temporal evolution of oxTMB concentrations at the early stage of the reactions catalyzed by HRP in dark at various pHs. The temperature of the bulk solutions was controlled at $25 \pm 1{ }^{\circ} \mathrm{C}$ using a water bath, and the $\mathrm{pH}$ of the reaction solutions was controlled using HAc-NaAc buffers. The initial concentrations of the Au785, $\mathrm{HRP}, \mathrm{H}_{2} \mathrm{O}_{2}$, and TMB were $180 \mathrm{fM}, 11 \mathrm{pM}, 568 \mathrm{mM}$, and $750 \mu \mathrm{M}$, respectively.
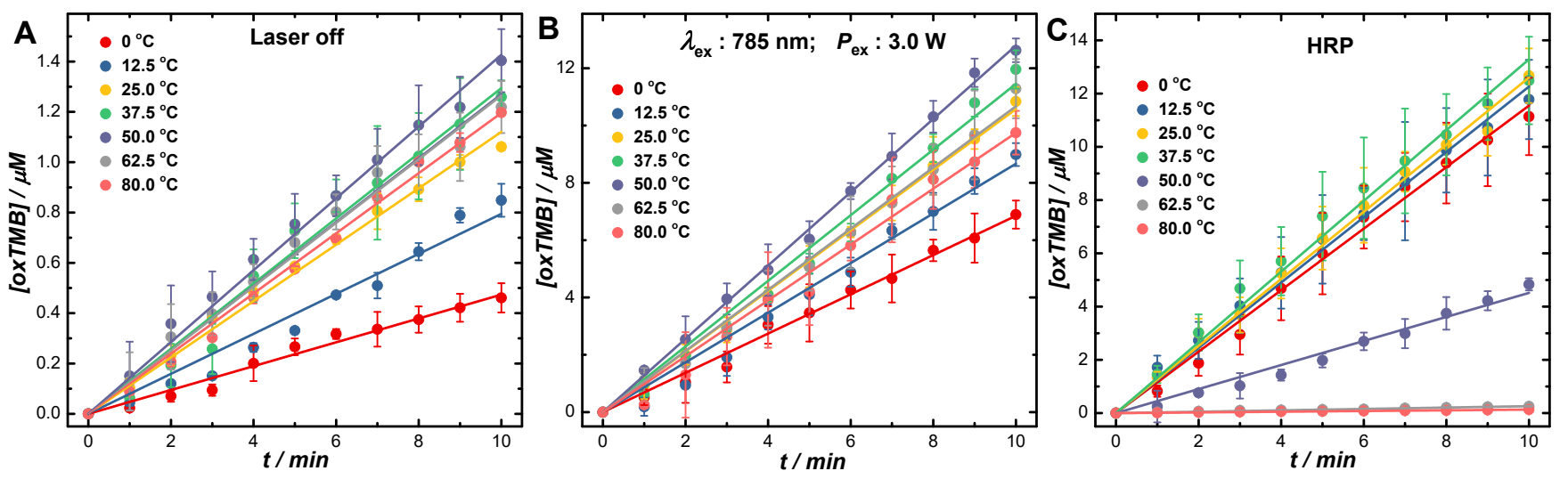

Figure S20. Temporal evolution of oxTMB concentrations at the early stage of the reactions catalyzed by Au-785 (A) in dark and (B) under laser illumination $\left(\lambda_{\mathrm{ex}}=785 \mathrm{~nm}, P_{\mathrm{ex}}=3.0 \mathrm{~W}\right)$ at various reaction temperatures. (C) Temporal evolution of oxTMB concentrations at the early stage of the reactions catalyzed by HRP in dark at various reaction temperatures. The temperatures of the bulk solutions were controlled using a water bath, and the $\mathrm{pH}$ of the reaction solutions was kept at 4.5. The initial concentrations of the Au785, $\mathrm{HRP}, \mathrm{H}_{2} \mathrm{O}_{2}$, and TMB were $180 \mathrm{fM}, 11 \mathrm{pM}, 568 \mathrm{mM}$, and $750 \mu \mathrm{M}$, respectively. 


\section{S4. References for Supporting Information}

1. Zhang, Q. F.; Large, N.; Nordlander, P.; Wang, H. Porous Au Nanoparticles with Tunable Plasmon Resonances and Intense Field Enhancements for Single-Particle SERS. J. Phys. Chem. Lett. 2014, 5, 370-374.

2. Zhang, Q. F.; Blom, D. A.; Wang, H. Nanoporosity-Enhanced Catalysis on Subwavelength Au Nanoparticles: A Plasmon-Enhanced Spectroscopic Study. Chem. Mater. 2014, 26, 5131-5142.

3. Villarreal, E.; Li, G. G.; Zhang, Q. F.; Fu, X. Q.; Wang, H. Nanoscale Surface Curvature Effects on Ligand-Nanoparticle Interactions: A Plasmon-Enhanced Spectroscopic Study of Thiolated Ligand Adsorption, Desorption, and Exchange on Gold Nanoparticles. Nano Lett. 2017, 17, 4443-4452.

4. Zhang, J.; Langille, M. R.; Personick, M. L.; Zhang, K.; Li, S. Y.; Mirkin, C. A. Concave Cubic Gold Nanocrystals with High-Index Facets. J. Am. Chem. Soc. 2010, 132, 14012-14014.

5. Li, G. G.; Lin, Y.; Wang, H. Residual Silver Remarkably Enhances Electrocatalytic Activity and Durability of Dealloyed Gold Nanosponge Particles. Nano Lett. 2016, 16, 7248-7253.

6. Pedireddy, S.; Lee, H. K.; Tjiu, W. W.; Phang, I. Y.; Tan, H. R.; Chua, S. Q.; Troadec, C.; Ling,

X. Y. One-Step Synthesis of Zero-Dimensional Hollow Nanoporous Gold Nanoparticles with Enhanced Methanol Electrooxidation Performance. Nat. Commun. 2014, 5, 4947.

7. Trasatti, S.; Petrii, O. A. Real Surface-Area Measurements in Electrochemistry. J. Electroanal. Chem. 1992, 327, 353-376.

8. Zhang, X. Q.; Gong, S. W.; Zhang, Y.; Yang, T.; Wang, C. Y.; Gu, N. Prussian Blue Modified Iron Oxide Magnetic Nanoparticles and Their High Peroxidase-Like Activity. J. Mater. Chem. 2010, 20, 5110-5116.

9. Ainsworth, S. Michaelis-Menten Kinetics. In Steady-State Enzyme Kinetics, Ainsworth, S., Ed. Macmillan Education UK: London, 1977; pp 43-73.

10. Orishchin, N.; Crane, C. C.; Brownell, M.; Wang, T.; Jenkins, S.; Zou, M.; Nair, A.; Chen, J. Rapid Deposition of Uniform Polydopamine Coatings on Nanoparticle Surfaces with Controllable Thickness. Langmuir 2017, 33, 6046-6053. 